\title{
Solvation Dynamics of DCM in Lipid
}

\author{
Samir Kumar Pal, Dipankar Sukul, Debabrata Mandal, and Kankan Bhattacharyya* \\ Physical Chemistry Department, Indian Association for the Cultivation of Science, \\ Jadavpur, Calcutta 700 032, India
}

Received: September 20, 1999; In Final Form: January 10, 2000

\begin{abstract}
Solvation dynamics of 4-(dicyanomethylene)-2-methyl-6(p-dimethylaminostyryl)-4H-pyran (DCM) in dimyristoylphosphatidylcholine (DMPC) vesicles in water is studied by using the picosecond time-resolved Stokes shift technique. In unilamellar DMPC vesicles, DCM exhibits wavelength-dependent fluorescence decays with a fast decay at the blue end and a slow decay preceded by a distinct growth at the red end of the emission spectrum. The solvation dynamics of DCM in DMPC vesicles is found to be biexponential with two components $230 \mathrm{ps}(40 \%)$ and $1.6 \mathrm{~ns}(60 \%)$ and is attributed to the relaxation of the water molecules inside the water pool of DMPC vesicles.
\end{abstract}

\section{Introduction}

One of the longstanding goals of chemistry and biology is to elucidate the dynamics of various processes occurring in complex biological assemblies. Most processes in biological systems occur in a confined region having dimensions of a few nanometers. Confinement in a small space and the severely altered local properties markedly modify dynamics of many chemical processes. As a result, chemistry in biological and confined environments is often very different from that in bulk water. The behavior of water in such a confined environment plays a fundamental role in many natural processes. In recent years several groups have studied solvation dynamics and dielectric relaxation of water in many organized and constrained media such as cyclodextrin, ${ }^{1}$ proteins, ${ }^{2}$ microemulsions, ${ }^{3-6}$ micelles, ${ }^{7}$ water surface, ${ }^{8}$ and lipid vesicles. ${ }^{9}$ Of these, the study of lipid vesicles is most relevant to understanding the behavior of water in a biological cell. In a vesicle, an aqueous volume is surrounded by a bilayer of surfactants and is dispersed in bulk water. ${ }^{10-12}$ In a recent paper, we have demonstrated that for coumarin 480 (C-480) in dimyristoylphosphatidylcholine (DMPC) lipid the solvation dynamics exhibits a component of 600 ps. ${ }^{9}$ This is substantially slower than the subpicosecond $(0.3 \mathrm{ps})$ solvation dynamics exhibited in bulk water. ${ }^{\text {la }}$ In the present work we report on the ultrafast dynamics of the laser dye 4-(dicyanomethylene)-2-methyl-6(p-dimethylaminostyryl)-4H-pyran (DCM, I) in DMPC vesicles. Recent femtosecond studies reveal

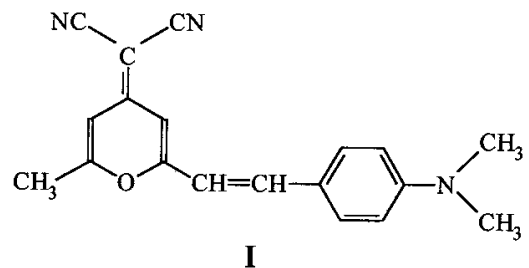

that solvation dynamics of DCM in methanol exhibits an inertial component on the 100 fs time scale and another of a few picoseconds. ${ }^{13,14}$ The structure of DCM suggests the possibility of various ultrafast processes, such as isomerization about the

*E-mail: pckb@mahendra.iacs.res.in. Fax: (91)-33-473-2805. olefinic double bond, twisted intramolecular charge transfer (TICT), apart from the ordinary solvation dynamics. Because of the TICT process, the excited-state dipole moment of DCM $(23.6 \mathrm{D})$ is very much higher than that in the ground state. ${ }^{13,14}$ It is obviously of interest to find out how these ultrafast processes are affected in organized assemblies. The TICT process is usually slowed in various organized assemblies, ${ }^{15}$ and thus it is important to know whether the TICT process is retarded sufficiently to exhibit dual emission. In a recent work, ${ }^{16}$ we studied DCM in AOT microemulsions. The microemulsions are basically nanometer sized water droplets surrounded by a surfactant layer and dispersed in bulk $n$-heptane. We observed that DCM does not exhibit dual emission in AOT microemulsions and exhibits only the TICT emission band. ${ }^{16}$ Furthermore, it is observed ${ }^{16}$ that in AOT microemulsions DCM displays slow solvation dynamics with an average solvation time of $1.23 \mathrm{~ns}$. In the present work, we report on the photophysical processes of DCM in DMPC lipids.

\section{Experimental Section}

DCM (laser grade, Exciton) and DMPC (Sigma) were used as received. Absorption and emission spectra were recorded by a JASCO 7850 and a Perkin-Elmer 44B instruments, respectively. For lifetime measurements, the sample was excited at $300 \mathrm{~nm}$ with the second harmonic of a cavity dumped rhodamine 6G dye laser (Coherent 702-1) pumped by a cw mode locked Nd:YAG laser (Coherent Antares 76s). The emission was detected at magic angle polarization, using a Hamamatsu MCP photomultiplier (2809U). The full width at half-maximum of the instrument response at $300 \mathrm{~nm}$ is $\approx 80 \mathrm{ps}$. Fluorescence decays were deconvoluted by using global lifetime analysis software (PTI).

The lipid was prepared following the methanol injection method. ${ }^{10 \mathrm{c}, \mathrm{d}}$ Two milligrams of DMPC was dissolved in $50 \mu \mathrm{L}$ of a solution of DCM in methanol. Using a microliter syringe, the whole solution was rapidly injected into tris buffer of $\mathrm{pH}$ 7.4. In this way the methanol is diluted almost instantaneously in water and the phospholipid molecules are dispersed evenly throughout the medium. The mixture is kept for $1 \mathrm{~h}$ at $30^{\circ} \mathrm{C}$. The concentration of the final solution of DMPC was $1 \mathrm{mM}$ 

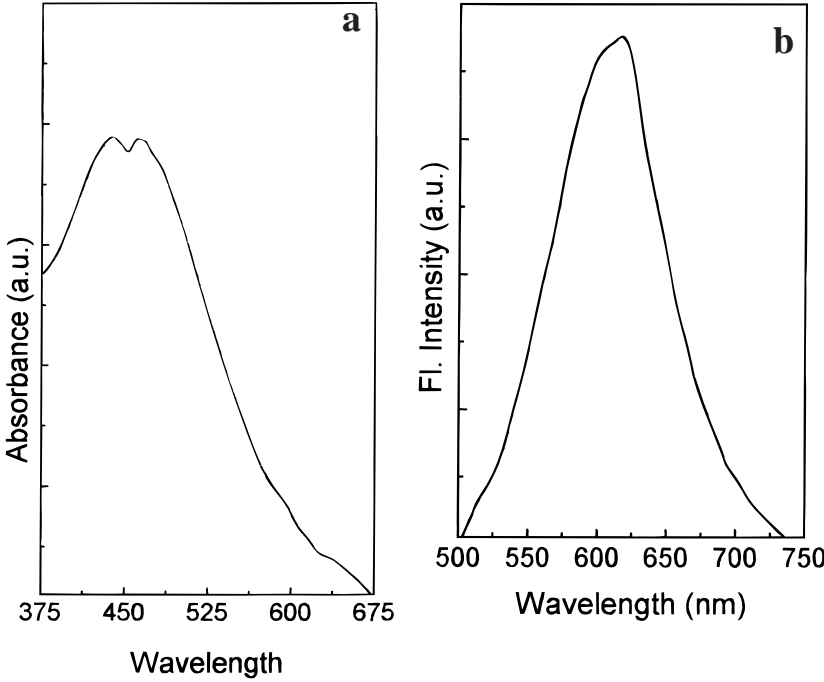

Figure 1. (a) Absorption spectrum of DCM in $1 \mathrm{mM}$ DMPC lipid. (b) Emission spectrum of DCM in $1 \mathrm{mM}$ DMPC lipid.

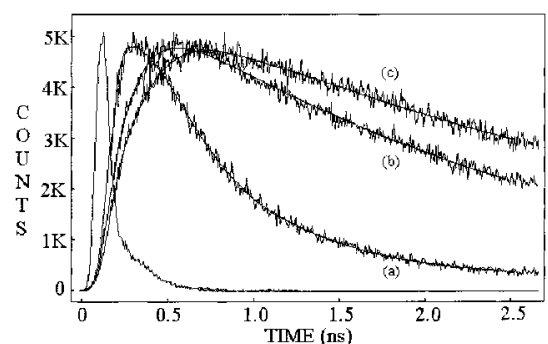

Figure 2. Fluorescence decays of DCM in $1 \mathrm{mM}$ DMPC lipid at (a) $520 \mathrm{~nm}$, (b) $620 \mathrm{~nm}$, and (c) $700 \mathrm{~nm}$.

and DCM concentration was $1 \times 10^{-5} \mathrm{M}$ so that the probe-tolipid ratio was $1: 100$. This procedure yields a high proportion of small unilamellar vesicles of diameter ca. $50 \mathrm{~nm} .{ }^{10 \mathrm{c}, \mathrm{d}}$ Emission quantum yields were obtained by using the reported quantum yield (0.44) of DCM in methanol. ${ }^{14 a}$ All steady-state and timeresolved measurements were done at $26^{\circ} \mathrm{C}$, i.e., above the gel transition temperature of DMPC $\left(23{ }^{\circ} \mathrm{C}\right) .{ }^{10}$

\section{Results}

3.1. Steady-State Spectra. In the DMPC lipid, DCM exhibits an absorption spectrum with two peaks at 445 and $470 \mathrm{~nm}$ (Figure 1a) and a strong emission band at $615 \mathrm{~nm}$ with $\phi_{\mathrm{f}}=$ 0.24 (Figure 1b). It is readily seen that DCM does not show dual emission in DMPC. The emission maximum of DCM exhibits a red shift with increase in solvent polarity, from 530 $\mathrm{nm}$ in $n$-heptane to $620 \mathrm{~nm}$ in methanol. DCM is insoluble in water and hence, the steady-state spectral properties of DCM in lipid could not be compared with those in water. However, the observed emission maximum of DCM in lipid is similar to that in methanol.

3.2. Time-Resolved Studies. The fluorescence decay of DCM in DMPC exhibits a marked wavelength dependence. At the blue end a fast decay is observed, while at the red end the decay is preceded by a growth (Figure 2). Such a wavelength dependence is typical of molecules undergoing solvation dynamics. ${ }^{17}$ From the parameters of best fit for the emission decays and using the steady-state emission spectra, time-resolved emission spectra (TRES, Figure 3) have been constructed following the procedure described by Fleming and Maroncelli. ${ }^{17 \mathrm{c}}$ The solvation dynamics is described by the response function $C(t)$ which is defined as

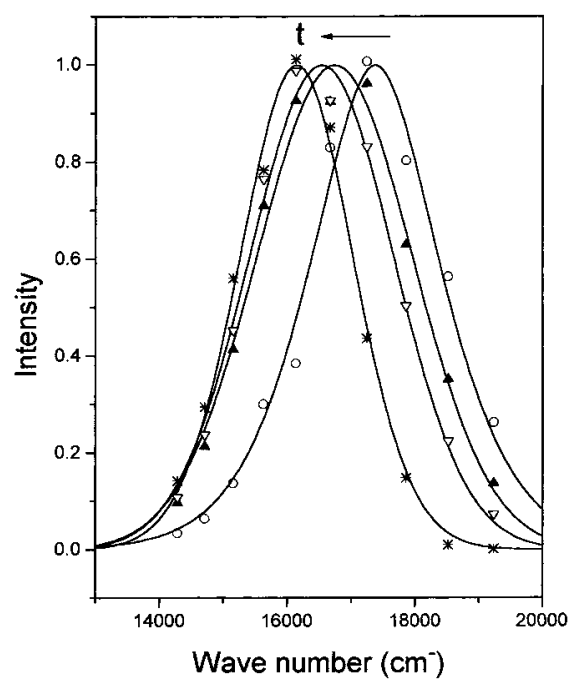

Figure 3. Time-resolved emission spectra of DCM in $1 \mathrm{mM}$ DMPC lipid at 0 ps $(\bigcirc), 500$ ps $(\mathbf{\Lambda}), 1000$ ps $(\nabla)$, and 10000 ps $(*)$.

$$
C(t)=\frac{v(t)-v(\infty)}{v(0)-v(\infty)}
$$

The decay of $C(t)$ is shown in Figure 4. The decay parameters of $C(t)$ are summarized in Table 1 . It is readily seen that the total Stokes shift $(v(0)-v(\infty))$ is $1250 \mathrm{~cm}^{-1}$ and the decay of $C(t)$ is biexponential with two components 230 ps $(40 \%)$ and $1600 \mathrm{ps}(60 \%)$ leading to an average solvation time, $\left\langle\tau_{\mathrm{s}}>\right.$ $\left(=a_{1} \tau_{1}+a_{2} \tau_{2}\right)$ of $1050 \mathrm{ps}$.

\section{Discussion}

As noted earlier, DCM does not exhibit dual emission in the DMPC lipid. The observed emission at $615 \mathrm{~nm}$ is very close to that of the TICT band of DCM in polar solvents. ${ }^{13,14}$ This indicates that the TICT process of DCM remains ultrafast in the lipid.

The time-dependence Stokes shift (TDSS) indicates that DCM exhibits a $\Delta v=1250 \mathrm{~cm}^{-1}$ in the DMPC lipid. It may be noted that recent femtosecond studies indicate that DCM exhibits a $\Delta v=3800 \mathrm{~cm}^{-1}$ in methanol and $2400 \mathrm{~cm}^{-1}$ in ethylene glycol. ${ }^{13 \mathrm{~b}, \mathrm{c}}$ Though the total Stokes shift depends on solvent, in our apparatus with limited time resolution ( $~ 80 \mathrm{ps})$ one cannot eliminate the possibility of missing a large part of solvation which occurs in the femtosecond time scale. ${ }^{13,14}$ Despite this limitation it is evident that the solvation dynamics of DCM in DMPC exhibits a component of about $1 \mathrm{~ns}$, which is substantially slower than those obtained in the femtosecond studies. ${ }^{13,14}$

In a lipid vesicle, there are three possible locations of the probe, bulk water, inner water pool, and bilayer. Since DCM is insoluble in water the possibility of DCM in bulk water is ruled out. In a hydrocarbon the emission quantum yield of DCM is extremely low $(0.01)$ and the lifetime is very short $(<50 \mathrm{ps}) .{ }^{16}$ Further, in $n$-heptane the emission maximum of DCM is very much blue-shifted to $530 \mathrm{~nm}$ and the decay does not exhibit a wavelength dependence. ${ }^{16}$ Thus the DCM molecules staying in the "dry" hydrocarbon-like bilayer is not expected to contribute to the observed solvation dynamics. Thus the solvation dynamics appears to be due exclusively to the DCM molecules in the inner water pool. The observed solvation time of $1 \mathrm{~ns}$ is very similar to that of DCM obtained in the water pool of the microemulsion $^{16}$ and is very close to the 600-ps component reported for DMPC using C-480 as a probe. ${ }^{9}$ This indicates solvent relaxation time of the water molecules confined by the lipid bilayer is $1 \mathrm{~ns}$. This is substantially slower than the 


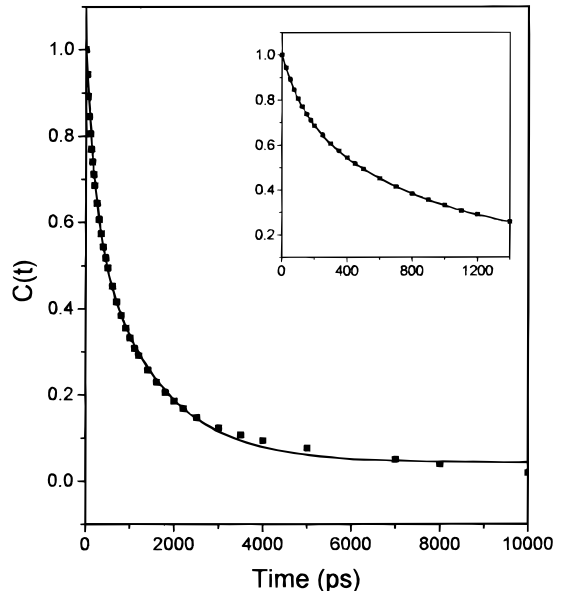

Figure 4. Decay of response function, $C(t)$ of DCM in $1 \mathrm{mM}$ DMPC lipid. The points denote the actual values of $C(t)$ and the solid line denotes the best fit to a biexponential decay. The decay of the initial portion is given in the inset.

TABLE 1: Decay Parameters of $C(t)$ of DCM in DMPC Lipid

\begin{tabular}{cccccc}
\hline$\Delta \nu, \mathrm{cm}^{-1}$ & $a_{1}$ & $\tau_{1}, \mathrm{ps}$ & $a_{2}$ & $\tau_{2}, \mathrm{ps}$ & $<\tau_{\mathrm{s}}>,{ }^{a} \mathrm{ps}$ \\
\hline 1250 & 0.40 & 230 & 0.60 & 1600 & 1050 \\
$a<\tau_{\mathrm{s}}>=a_{1} \tau_{1}+a_{2} \tau_{2}$. & & & &
\end{tabular}

solvation dynamics in ordinary water. ${ }^{1 \mathrm{a}, 17 \mathrm{a}}$ Kaatze and coworkers ${ }^{18}$ reported that lipid vesicles exhibit two prominent dielectric relaxation time $\left(\tau_{\mathrm{D}}\right)$ in 10 and $0.1 \mathrm{~ns}$ time scales. They assigned the 10-ns relaxation to the solvent relaxation time. Since in the continuum model ${ }^{17 \mathrm{~b}}$ solvation time, $\tau_{\mathrm{S}}=\left(\epsilon_{\infty} / \epsilon_{0}\right) \tau_{\mathrm{D}}$, if one assumes $\epsilon_{\infty}$ of the inner water pool same as that of water, i.e., $5,{ }^{17 b}$ and $\epsilon_{0}$ is of the inner water pool same as that of methanol $(\sim 40)$, one immediately calculates a solvation time of $(5 / 40) \times 10 \mathrm{~ns}$, i.e., $1.25 \mathrm{~ns}$, which is remarkably close to the observed solvation time of $1 \mathrm{~ns}$. The role of methanol appears to be minor because the overall concentration of methanol added is about $2 \%$, most of which should evaporate during preparation of the lipid. Further, the solvation dynamics of $\mathrm{DCM}^{13,14}$ and other probes ${ }^{19}$ in methanol occurs with an inertial component in the 100 -fs time scale and another in the $<10$-ps time scale. Thus the observed nanosecond dynamics cannot be because of traces of methanol. Again, the polarity of the inner pool for lipids prepared by the methanol injection method is similar to that of sonicated unilamellar vesicles. ${ }^{9}$

\section{Conclusion}

The present work shows that DCM does not exhibit dual emission in the DMPC lipid. This suggests that the TICT process of DCM remains ultrafast in lipids. The solvation dynamics indicate that in the inner water pool the water molecules relax on a 1-ns time scale. The solvation dynamics studied is consistent with the previous dielectric relaxation studies in lipids. ${ }^{18}$ Since the water molecules inside the water pool of vesicles resemble those in biological cells, this study demonstrates that the dynamics of biological water molecules is substantially slower than that of ordinary water molecules.

Acknowledgment. Thanks are due to Council of Scientific and Industrial Research (CSIR) and Department of Science and
Technology, Government of India for generous research grants. S.K.P., D.S. and D.M. thank CSIR for awarding fellowships.

\section{References and Notes}

(1) (a) Vajda, S.; Jimenez, R.; Rosenthal, S. J.; Fidler, V.; Fleming, G. R.; Castner, E. W., Jr. J. Chem. Soc., Faraday Trans. 1995, 91, 867. (b) Nandi, N.; Bagchi, B. J. Phys. Chem. 1996, 100, 13914.

(2) (a) Nandi, N.; Bagchi, B. J. Phys. Chem. B 1997, 101, 10954. (b) Nandi, N.; Bagchi, B. J. Phys. Chem. A 1998, 102, 8217. 3775 .

(3) Lundgren, I. S.; Heitz, M. P.; Bright, F. V. Anal. Chem. 1995, 67,

(4) (a) Sarkar, N.; Das, K.; Datta, A.; Das, S.; Bhattacharyya, K. J. Phys. Chem. 1996, 100, 10523. (b) Das, S.; Datta, A.; Bhattacharyya, K. J. Phys. Chem. A 1997, 101, 3299. (c) Mandal, D.; Datta, A.; Pal, S. K.; Bhattacharyya, K. J. Phys. Chem. B 1998, 102, 9070.

(5) (a) Willard, D. M.; Riter, R. E.; Levinger, N. E. J. Am. Chem. Soc. 1998, 120, 4151. (b) Riter, R. E.; Undiks, E. P.; Levinger, N. E. J. Am. Chem. Soc. 1998, 102, 2705. (c) Riter, R. E.; Undiks, E. P.; Kimmel, J. R.; Pant, D. D.; Levinger, N. E. J. Phys. Chem. B 1998, 102, 7931. (d) Shirota, H.; Horie, K. J. Phys. Chem. B 1999, 103, 1437.

(6) (a) Cho, C. H.; Chung, M.; Lee, J.; Nguyen, T.; Singh, S. Vedamuthu, M.; Yao, S.; Zhu, S.-B.; Robinson, G. W. J. Phys. Chem. B 1995, 99, 7806. (b) Zhu, S.-B.; Singh, S.; Robinson, G. W. Adv. Chem. Phys. 1994, 85, 627.

(7) (a) Sarkar, N.; Datta, A.; Das, S.; Bhattacharyya, K. J. Phys. Chem. 1996, 100, 15483. (b) Datta, A.; Mandal, D.; Pal, S. K.; Bhattacharyya, K. J. Mol. Liq. 1998, 77, 121.

(8) Zimdars, D.; Dapad, J. I.; Eisenthal, K. B.; Heinz, T. F. Chem. Phys. Lett. 1999, 301, 112.

(9) Datta, A.; Pal, S. K.; Mandal, D.; Bhattacharyya, K. J. Phys. Chem. $B$ 1998, 102, 6114.

(10) (a) Pasenkiewicz-Gierula, M.; Takaoka, V.; Miyagawa, H.; Kitamura, K.; Kusumi, A. J. Phys. Chem. A 1997, 101, 3677. (b). de Haas, K. H.; Blom, C.; van der Ende, D.; Devits, M. H. G.; Haveman, B.; Mellema, J. Langmuir 1997, 13, 6658. (c) "Liposomes: A practical approach"; New, R. R. C., Ed.; Oxford University Press: Oxford, England, 1990; p 63. (d) Stryer, L. In Biochemistry, 4th ed.; Freeman: New York, 1998; p 271.

(11) (a) Demochenko, A. P.; Ladokhin, A. S. Eur. Biophys. J. 1988, 15, 569. (b) Chattopadhyay, A.; Mukherjee, S. Biochemistry 1993, 32, 3804.

(12) (a) Cassol, R.; Ge, M.-T.; Ferrarini, A.; Freed, J. H. J. Phys Chem. B 1997, 101, 8782. (b) Sung-Suh, M. M.; Kevan, L. J. Phys Chem. A 1997, 101, 1414. (c) Jutila, A.; Kinnunen, P. K. J. J. Phys Chem. B 1997, 101, 7635. (d) Srivastava, A.; Eisenthal, K. B. Chem. Phys. Lett. 1998, 292, 345.

(13) (a) Gustavsson, T.; Baldacchino, G.; Mialocq, J.-C.; Pommeret, S. Chem. Phys. Lett. 1995, 236, 587. (b) van der Meulen, P.; Zhang, H.; Jonkman, M.; Glasbeek, M. J. Phys. Chem. 1996, 100, 5367. (c) Zhang, H.; Jonkman, A. M.; van der Meulen, P.; Glasbeek, M. Chem. Phys. Lett. 1994, 224, 551. (d) Jonkman, M.; van der Meulen, P.; Zhang, H.; Glasbeek, M. Chem. Phys. Lett. 1996, 256, 21.

(14) (a) Easter, D. C.; Baronavski, A. P. Chem. Phys. Lett. 1993, 201, 153. (b) Mayer, M.; Mialocq, J.-C. Opt. Commun. 1987, 64, 264. (c) HsingKang, Z.; Ren-Lan, M.; Er-pin, N.; Chu, G. J. Photochem. 1985, 29, 397. (d) Retting, W.; Majenz, W. Chem. Phys. Lett. 1989, 154, 335. (e) Gilabert, E.; Lapouyade, R.; Rulliere, C. Chem. Phys. Lett. 1988, 145, 262.

(15) (a) Bhattacharyya, K.; Chowdhury, M. Chem. Rev. 1993, 93, 507. (b) Grabowski, Z. R. Pure Appl. Chem. 1993, 65, 1751. (c) Datta, A.; Mandal, D.; Pal, S. K.; Bhattacharyya, K. J. Phys. Chem. B 1997, 101, 10221.

(16) Pal, S. K.; Mandal, D.; Sukul, D.; Bhattacharyya, K. Chem. Phys. Lett. 1999, 312, 178.

(17) (a) Jimenez, R.; Fleming, G. R.; Kumar, P. V.; Maroncelli, M. Nature 1994, 369, 471. (b) Maroncelli, M. J. Mol. Liq. 1993, 57, 1. (c) Maroncelli, M.; Fleming, G. R. J. Chem. Phys. 1987, 86, 6221.

(18) (a) Kaatze, U. Phys. Med. Biol. 1990, 35, 1663. (b) Kaatze, U. Pros. Colloid Polym. Sci. 1980, 67, 117. (c) Kaatze, U.; Henze, R. Ber. Bunsen-Ges. Phys. Chem. 1980, 84, 1102.

(19) (a) Kahlow, M. A.; Jarzeba, W.; Kang, T. J.; Barbara, P. F. J. Chem. Phys. 1988, 90, 151. (b) Horng, M. L.; Gardecki, J. A.; Papazyan, A.; Maroncelli, M. J. Phys. Chem. 1995, 99, 17311. (c) Shirota, H.; Pal, H.; Tominaga, K.; Yoshihara, K. J. Phys. Chem. 1996, 100, 14575. (d) Gustavsson, T.; Cassara, L.; Gulbinas, V.; Gurzadyan, G.; Mialocq, J.-C.; Pommeret, S.; Sorgives, M.; van der Meulen, P. J. Phys. Chem. A 1998, $102,4229$. 